19. United States Department of Agriculture, Agricultural Statistics 2004. Table 3-51

20. Zhan S., Ho S.C. Meta-analysis of the effects of soy protein containing isoflavones on the lipid profile // Am J Clin Nutr 2005, 81: 397- 408

\title{
DOI 10.22448/AMJ.2017.3.34-35 DISCUSSION ON THE THEORY OF WUII POWDER IN TREATING UTERINE FIBROIDS
}

\author{
Fu Qiang1, Hu Xiaoyang2, Pang Yuhang1 \\ 1.Heilongjiang University of Traditional Chinese Medicine,Haerbin,China 2. First Affiliated Hospital Heilongjiang Univer- \\ sity of Chinese Medicine, Haerbin, China
}

Abstract: uterine myoma is the most common benign tumor of female reproductive organs, the prevalence of up to $51.2 \% \sim 60 \%$. The etiology of this disease is unclear, is difficult to cure the disease of department of gynaecology. Five jisan as treatments for cold, wet, qi, blood, phlegm, five kinds of stagnants, based on the theory of traditional Chinese medicine for five jisan theorizing about the treatment of uterine fibroids.

Key words: Wuji Powder; uterine fibroids; abdominal mass

Uterine fibroids are the most common benign tumors in women's reproductive health. They are prone to 30-50 year old women. Uterine fibroidsnot as malignant as a direct harm to the lives of patients, but the symptoms caused by a serious threat to women's health.There is no name of uterine fibroids in traditional Chinese medicine(TCM), but according to its clinical manifestations should be attributed to the "Zhengjia" ,"stone Zhengjia "and other areas[1].Wuji Powder is recorded in <Prescriptions People's Welfare Pharmacy>, which is treat with cold, wet, qi, blood and phlegm. This article will discuss from the Zhengjia etiology and pathogenesis and the composition and compatibility of the Wuji Powder.

\section{Knowledge of uterine fibroids in TCM}

According to the clinical symptoms: abdominal mass, menstrual extension, the amount of excessive, abdominal pain and other symptoms, will be attributed to the "tumor" category. <Ling Shu>:"Stone addiction was born in the womb, the cold qi in the sub-door, sub-door occlusion, qi can not pass, blood stasis shrimp to flow, days to benefits, like have a baby....",<Lady's prescription>:"The abdomen of the woman's blood, by the menstrual closure, or postpartum blood is not complete, or cold and stagnant, for a long time without the accumulation of scar Zheng Jia carry on."Blood chamber is open, uterine emptiness, wind cold dampness evil virtual invasion of the uterus, stagnation of blood, cold condensate blood stasis; or intercourse section, damage the blood cells, or worry about spleen, or Yu injury liver, can lead to blood stasis, tied to the cells, gradually become blocks, increasingly large uterine fibroids. [2]<Fuke Yuchi> emphasize the relationship between emotional abnormality and morbidity.In short, blood stasis is the pathogenesis of fibroids critical pathogenesis, or qi stagnation, or phlegm stasis type, or Qi and blood stasis. Disease occurs in the process, often dirty organs dysfunction, especially in the liver, spleen, kidney dysfunction more common.

\section{The group analysis of Wuji Powder}

Wuji Powder scattered from the <Prescriptions People's Welfare Pharmacy>, composed by the Atractylodes, Magnolia, dried tangerine peel, Pinellia, Poria, licorice, ephedra, cassia twig, white peony root, angelica, Chuanxiong, dried ginger, Citrus aurantium, Angelica and other 15-drug.Atractylodes, Magnolia, dried tangerine peel, licorice is Pingwei Powder for the transport of spleen dampness digestion. Dried tangerine peel, Pinellia, Poria, licorice as the main phlegm of the Erchen Tang.There is governance of the sun table card Guizhi soup; another rule of phlegm Ling Guigu Gan Tang; kidney disease Ling Jiang Gan Gan Tang.Siwu decoction to remove Rehmannia, with the blood of the meridian of the power; there ephedra Guixi Xin Wen published to scatter cold;Dried ginger, Guizhi, Citrus aurantium, Magnolia temperature in the qi, Dried tangerine peel, Pinellia ternata and ephedra, Campanulaceae Xuanfei phlegm; Ephedra, cassia twig, dried ginger, white peony root, angelica, licorice with the meaning of the side of the soup, all drugs combined, can eliminate the table, in the gas, blood cold wet.[3]Because this can cure cold, wet, qi, blood, sputum five kinds of weakness and the dosage form and powder mainly to take "scattered people also scattered" effect, named Wuji powder.

\section{Theoretical Analysis of Wuji Powder in Treating Uterine}

Uterine fibroids Zhengjia common gynecological diseases, the main cause of qi stagnation, blood stasis and phlegm pattern three causes. The main pathogenesis of the viscera and bwels dysfunction for the woman, resulting in qi deficiency with blood stasis, and phlegm-dampness generated, and then accumulate in the belly, in the course of the disease, the three each other cause and effect, mutual transformation, complex. In the past, treatment is often based on activate blood and resolve stasis, and the disease at different stages of its etiology is not the same, and the disease often changes in the pathogenesis, if the prescription medication can not take care of both, it is difficult to achieve therapeutic effect. [4] The disease due to cold in the middle form dampness, dampness agglomerate is phlegm, phlegm-dampness caused qi stagnation and blood stasis.So the treatment is used to activate blood and resolve stasis, move qi to relieve pain, warm the meridian to dissipate cold,fortify the spleen and drain dampness. Until remove the cold, resolve phlegm-dampness, spleen yang back to the revitalization,harmony of qi and blood, the card that is cured.In the Wuji Powder, Ephedra, Angelica promote sweating to release the exterior, Herb, Chuan Park, dried tangerine peel, Zhigancao dry dampness to 34 Амурский медицинский журнал №3 (19) 2017 
fortify the spleen, Dried ginger, cinnamon warm the interior to dissipate cold,Pinellia, Poria dry dampness to resolve phlegm, Citrus aurantium, Campanulaceae move qi to soothe the chest, Angelica, Chuanxiong, white peony root activate blood to relieve pain.The whole party can dissipate cold, dispel dampness, regulate qi, activate blood, resolve phlegm. It is the best formula to treat the disease with cold, dampness, qi, blood, phlegm combined,especially uterine fibroids.

\title{
4.Conclusion
}

The pathogenesis of Zhengjia is complex, but the total pathogenesis are blood stasis due to qi stagnation and phlegm-dampness accumulation in the abdomen, form the disease. So the treatment principles are activate blood and resolve stasis, move qi to relieve pain, remove dampness to resolve phlegm.Wuji Powder is a exterior and interior-yin and yang formula to treated cold, dampness, qi, blood, phlegm combined.Therefore, the treatment of the disease can be adjusted according to different mains of the drug side of the drug, the syndrome both, the effect is significant.

\section{Reference:}

[1] Yan Weina, Du Dongqing, Yang Jianbing. Chinese medicine treatment of uterine fibroids [J]. Modern Journal of Traditional Chinese Medicine, 2008,06: 74-75.

[2] Zhang Yingqi. Guizhi Fuling pill treatment Zhengjia (uterine fibroids) of the literature [D]. Beijing University of Traditional Chinese Medicine, 2006.

[3] Dong Fei Xia. Five powder scattered clinical application [N]. Chinese Journal of Traditional Chinese Medicine, 2004-04-01.

[4] Xu Wenting, He Guixiang. Uterine fibroids Chinese medicine research overview [J]. Journal of Liaoning University of Traditional Chinese Medicine, 2010,10: 205-207.

UDC 616 DOI 10.22448/AMJ.2017.3.35-36

\section{STUDY OF THE UPPER RESPIRATORY TRACT BY THE METHOD OF FREEZING CHIPPING}

\author{
Gorbunov M.M., Tseluyko S.S., Zinoviev S.V.
}

Amur State Medical Academy, Blagoveshchensk, Russian Federation

Summary Ratsweresubjected toinhalationfor 3 hours 5 daysapparatusVulcan3. Usingscanningelectronmicroscopymethod offreezingchippingrevealedsignificantchangestaking placeonthesurfaceofthetrachea. Thisisaperiod ofadaptationvoltage atwhichthesurfaceiswavy, sharpness ofthecilia ofciliatedepithelium, gobletcellsareformed, mucusandcellularelements.

Key words: method of freezing cleaving, during stress adaptation

Materials and methods The study was conducted on male rats weighing unit Rodecia 200-300 grams of 20 pieces. Rats were placed in a special chamber at ambient temperature and $+5 S$ inhalations moist air for 3 hours to 5 days apparatus Vulcan-3. Then the rats were sacrificed in order to obtain a complex of the respiratory system [2]. Study of the method was carried out using freeze - spalling, wherein investigated mucosal surface of the trachea and bronchi and alveolar walls of the lungs [3]. The material was placed in liquid nitrogen -196Co and frozen within 10 minutes. Thereafter, the bodies cleaved sharp razor blade and placed on a freezing table of a scanning electron microscope Hitachi [4].

Results and discussion In the study of the surface of the trachea is noteworthy change in the topography of its mucosa. Found a large accumulation of undulating cilia over the entire surface of the mucous membrane of the trachea. Cilia are clearly distinguishable, have the correct shape, and each of them separated from each other. Against the background of various ciliary epithelium allocated tops exaggerated apical pole of goblet cells lacking microvilli, these formations are pushing the cilia of ciliated cells. The secretory activity of goblet cells is accompanied by mucus on the surface epithelium of the destruction of the apical pole.

Consequently, it is often found in the mucus residues of cytoplasmic components. This causes that adhesion of slime causes the cilia and are in some parts thereof are arranged in groups in the study are hardly distinguishable electron microscope. Picture of the state of goblet cells creates the impression of a violation of the secretory cycling as all slime cells are in the same stage - discharge. The apical surface of the ciliated cells in a hypersecretion has many bulging. Raster terrain analysis of ciliated cells revealed a large number of mushroom-shaped protrusions.

Conclusions Thus, in the course of the experiments with the use of cold in combination with inhalation of moist air, we observe the voltage adaptation period, this leads to a change in the qualitative composition of the epithelium of tracheal mucosa [1]. When pathological changes which affect in varying degrees, all elements of the surface epithelium, primarily ciliary cells. We see that the structure of fibrillar apparatus changes the mucosal surface becomes wavy and sometimes loses clarity. Also, there are areas with a predominance of ciliated epithelium goblet cells devoid of microvilli forming a dome. The secretory activity of goblet cells is accompanied by mucus on the surface epithelium, causing a violation mukotsilliarnogo transport due to adhesion of the cilia in the future leads to a violation of shear-wave movement of the cilia. Our proposed method has advantages over other known methods. As we study the native properties of the mucous membrane of the trachea and its secrets. When the selected mode is not freezing phase crystallization of water characterizing the osmolarity of biological objects. Therefore, freezing in liquid nitrogen is the most physiologic method of research.

Bibliography 